\title{
Cost and effects of integrated care: a systematic literature review and meta-analysis
}

\author{
Stephen Rocks ${ }^{1} \oplus \cdot$ Daniela Berntson $^{1} \cdot$ Alejandro Gil-Salmerón $^{2} \cdot$ Mudathira Kadu $^{3} \cdot$ Nieves Ehrenberg $^{4}$. \\ Viktoria Stein $^{4} \cdot$ Apostolos Tsiachristas $^{1}$ (D)
}

Received: 11 September 2019 / Accepted: 30 June 2020 / Published online: 6 July 2020

(c) The Author(s) 2020

\begin{abstract}
Background Health and care services are becoming increasingly strained and healthcare authorities worldwide are investing in integrated care in the hope of delivering higher-quality services while containing costs. The cost-effectiveness of integrated care, however, remains unclear. This systematic review and meta-analysis aims to appraise current economic evaluations of integrated care and assesses the impact on outcomes and costs.

Methods CINAHL, DARE, EMBASE, Medline/PubMed, NHS EED, OECD Library, Scopus, Web of Science, and WHOLIS databases from inception to 31 December 2019 were searched to identify studies assessing the cost-effectiveness of integrated care. Study quality was assessed using an adapted CHEERS checklist and used as weight in a random-effects meta-analysis to estimate mean cost and mean outcomes of integrated care.

Results Selected studies achieved a relatively low average quality score of $65.0 \%( \pm 18.7 \%)$. Overall meta-analyses from 34 studies showed a significant decrease in costs $(0.94$; CI 0.90-0.99) and a statistically significant improvement in outcomes (1.06; CI 1.05-1.08) associated with integrated care compared to the control. There is substantial heterogeneity in both costs and outcomes across subgroups. Results were significant in studies lasting over 12 months (12 studies), with both a decrease in cost $(0.87$; CI $0.80-0.94)$ and improvement in outcomes $(1.15$; 95\% CI 1.11-1.18) for integrated care interventions; whereas, these associations were not significant in studies with follow-up less than a year.

Conclusion Our findings suggest that integrated care is likely to reduce cost and improve outcome. However, existing evidence varies largely and is of moderate quality. Future economic evaluation should target methodological issues to aid policy decisions with more robust evidence on the cost-effectiveness of integrated care.
\end{abstract}

Keywords Cost-effectiveness $\cdot$ Economic evaluation $\cdot$ Integrated care; meta-analysis

\section{Introduction}

Electronic supplementary material The online version of this article (https://doi.org/10.1007/s10198-020-01217-5) contains supplementary material, which is available to authorized users.

Apostolos Tsiachristas

apostolos.tsiachristas@ndph.ox.ac.uk

1 Health Economics Research Centre, Nuffield Department of Population Health, University of Oxford, Oxford, UK

2 Polibienestar Research Institute, Universitat de València, Valencia, Spain

3 Institute of Health Policy, Management and Evaluation, University of Toronto, Toronto, ON, Canada

4 International Foundation for Integrated Care, Oxford, UK
Governments across high-income countries are challenged to contain the relentless increase in health expenditure, which is partly driven by ageing populations and an associated increase in the prevalence of chronic disease [1]. This challenge is increasingly concerning as many health systems have become highly specialised, fragmented and poorly set to manage the growing burden of multimorbidity [2]. Increasing efficiency in care delivery by integrating health services has been proposed as a solution to healthcare budget issues [3-5]. Integrated care is an umbrella term that encompasses a diverse set of methods and models that facilitate improvement in patient experience through enhanced coordination and continuity of care [6,7]. As such, integrated care covers a wide range of treatment plans and organizational 
models, does not discriminate between diseases or target populations, and has varied consequences across different treatment areas [8]. The Triple Aim of integrated care is to improve population health, enhance user experience with care and reduce growing healthcare expenditure [9-12].

Despite pressure to assess the cost-effectiveness of integrated care interventions to appropriately inform decision makers of the potential financial benefits of moving towards new models of care [13], the evidence from economic evaluations has thus far been inconclusive [14-16]. This is widely attributed to the lack of reliable evidence summarized by Nolte and colleagues (2014) who identified the "quality of existing economic evaluations as the main impediment to arriving at robust evidence to inform decision making" [17]. Indeed, a recent systematic literature review showed that economic evaluations in integrated care had on average poor-to-moderate methodological quality [18]. In addition, the unclear definition of what constitutes integrated care and the large variation in the models of integration within and across geographic areas poses a challenge to the reliability and replicability of evaluation studies [19].

Currently, there is a dearth of meta-analyses of economic evaluations that compare integrated care models with conventional care; this evidence focuses on single conditions and certain models of integrated care [14, 20, 21]. In addition to the limited span of evidence, the overall impact of integrated care on costs and outcomes is still unclear. This paper aims to provide an up-to-date review of economic evaluations in integrated care and perform a meta-analysis to summarize the impact of integrated care on costs and outcomes.

\section{Methods}

\section{Search strategy}

We followed the search strategy of a recently published review of economic evaluations in integrated care that assessed their methodological quality [18]. For this updated literature review, we searched for eligible studies from inception to 31st December 2019 in the following databases: CINAHL, Database of Abstracts of Reviews of Effects (DARE), EMBASE, Medline/PubMed, the NHS Economic Evaluation Database (NHS EED), OECD Library, Scopus, Web of Science, and the World Health Organization library and information networks for knowledge database (WHOLIS).

Terms relating distinctly to the broad concepts of "integrated care" and "economic evaluation" were identified in relevant journals (International Journal of Integrated Care) and previous systematic reviews that reported on the costeffectiveness of integrated care [14]. Our search was also informed by terms used in the Integrated Care Search tool developed by the International Foundation for Integrated Care [22]. Frequently used expressions were then compiled to form the list of search terms, shown in Supplementary material Figure S1. Additionally, reference lists featured in key publications [17] (including systematic reviews, opinion pieces and editorials on integrated care) were hand-searched to identify any relevant articles that were otherwise missed $[23,24]$.

\section{Selection process and eligibility criteria}

All study titles and abstracts were added in an online citation manager tool, Mendeley. Study selection occurred in a twostep process based on the pre-set inclusion and exclusion criteria detailed below. First, titles and abstracts were screened by DB or AGS, and second, full text of selected studies were screened for final inclusion. To ensure consistency between the reviewers, AT screened the titles, abstracts, and full texts of $10 \%$ of the articles screened and selected by the other reviewers $[25,26]$. The target for inter-reviewer agreement was $95 \%$. When there was uncertainty over whether to exclude an article based upon understanding of its title and abstract the full text was read and its inclusion was discussed between DB, AGS and AT until a decision was made.

Inclusion criteria:

1 Articles describing the implementation, execution or evaluation of interventions or programs based on the most frequently used definition of integrated care: "funding, administrative, organisational, service delivery and clinical interventions designed to create connectivity, alignment and collaboration within and/or between the cure and care sectors" [27].

2 Articles including empirical economic evaluations as defined by Drummond and colleagues: "the comparative analysis, measurement, valuing and identification of alternative courses of actions in terms of their cost and consequences" [28].

Exclusion criteria:

1 Articles published in languages other than English.

2 Articles solely describing the concept of integrated care or the rationale behind its implementation without reporting on an actual intervention or practice.

3 Systematic reviews, dissertations, conference proceedings, opinion pieces, editorials and conference abstracts on the subject of integrated care. 


\section{Data abstraction and quality assessment}

Elements from the PICO model [29] and abstraction templates designed by Boland and colleagues [20] were adapted for the purpose of qualitative data abstraction; data included sample sizes, study origin, follow-up period, study design and objectives, patient and intervention characteristics, whilst also describing the perspective from which the economic evaluation was taken (Supplementary material Table S1).

A second template, designed as a checklist, was developed as a tool to assess the overall quality of each study and their economic evaluations. The Consolidated Health Economic Evaluation Reporting Standards (CHEERS) [30] and the health technology assessment of disease management programs (HTA-DM) [31] were consolidated into a list of 30 items upon which a binary scoring system acted to assess study strengths and weaknesses (Supplementary material Table S2). Each study was given a quality score to assess overall study quality. The quality score was a proportion with a maximum of $100 \%$ (highest possible quality), where the numerator is the sum of the binary scores (maximum of 30) divided by the denominator (30 minus any notapplicable scores).

A final template was designed to collate both qualitative and quantitative data regarding the outcome measures of treatment cost and effectiveness (see Supplementary material Tables S3 and S4). Principal information extracted included: currency; cost categories included in total cost; mean total cost of intervention and control; mean cost ratio; ICER, if included; measurement of quality of life; mean quality of life score; and mean quality of life ratio.

All templates were initially trialled on the data abstraction of four studies before use on all selected studies.

\section{Meta-analysis and statistical approaches}

Studies found to contain insufficient cost or outcome data during data extraction were excluded from the meta-analysis. Cost and outcome data extracted from the same studies were analyzed separately.

In the case of cost analyses, the ratio of final costs associated with the intervention and control group was calculated for each study. The use of a cost ratios annuls differences in measurement across studies, which means currency conversions, inflation compensations, and standardization of varying cost scales were not necessary. The majority of studies did not report any standard deviation nor standard error of the reported costs and outcomes; therefore, we followed similar examples in the literature and used the study quality scores generated to weigh studies in the meta-analysis [32, 33]. In this way, higher-quality studies were given greater weight and lower-quality, and therefore less reliable, studies less weight.

For the assessment of care effectiveness, the ratio of mean effectiveness scores associated with the intervention and control group was calculated. The use of a ratio helps account for disparities in the measures used to assess effectiveness of care in individual studies, facilitating the pooling and comparison of their data [34]. In the case of a lower effect score translating to a better clinical outcome, the effect ratio was inverted to reflect the appropriate change in outcome.

For observational studies that did not control for confounding factors at baseline, additional adjustments were undertaken to compensate for baseline differences when assessing costs (Eq. 1) and effects (Eq. 2).

$\frac{\text { Finalmeancostofintervention }}{\text { Finalmeancostofcontrol }} \times \frac{\text { Baselinemeancostofcontrol }}{\text { Baselinemeancostofintervention }}$

$\frac{\text { Finalmeaneffectofintervention }}{\text { Finalmeaneffectofcontrol }} \times \frac{\text { Baselinemeaneffectofcontrol }}{\text { Baselinemeaneffectofintervention }}$

Stata12.1 SE (StataCorp, Tx) was used when undertaking analyses. To calculate a weighted average ratio of mean cost and outcome, the data were pooled using a random-effects meta-analysis model based on the DerSimonian-Laird method [35]. The variety in patient characteristics, and hence true effect size, across studies justified the use of random-effects meta-analysis. Heterogeneity in the results was then illustrated by forest plots grouped into study- (1) design, (2) duration, (3) region and (4) type of integrated care intervention.

\section{Results}

\section{Search results}

The initial review included 44 scientific articles and the detail of the selection process is described elsewhere [18]. The review update yielded a total of 3094 articles, with two further articles $[23,24]$ identified from subsequent citation screening of relevant reference lists. Following removal of duplicates and screening of title and abstract, 45 eligible studies remained for full-text examination. Finally, three studies [36-38] from the update search were considered to follow all pre-set inclusion and exclusion criteria and included in the final quality assessment and meta-analysis. Combining the initial and update reviews, 47 studies were brought forward for the quality assessment and data extraction. Of those, 13 studies were excluded from the meta-analysis due to inadequate cost and outcome reporting [39-51]. The reported costs and outcomes of 34 studies were used in 
the meta-analyses [24, 36-38, 52-80]. The adapted PRISMA flowchart of the review update is presented in Fig. 1 and follows Cochrane guidance for updated reviews [81].

\section{Study description}

There were sixteen (34\%) randomized control trials (RCT), twelve $(26 \%)$ cluster RCTs, ten $(21 \%)$ pre-post cohort studies, eight $(17 \%)$ cohort studies and one (2\%) cross-sectional study included (Supplementary material Table S1). Twentyfour $(51 \%)$ of these studies originated from Europe, whilst $16(34 \%)$ studies were conducted in North America, three $(6 \%)$ in Africa, two (4\%) in Asia, and two (4\%) in Australia. Study observation period varied from three to 36 months, excluding one cross-sectional study that assessed a large population [54]. Thirty-one of the selected studies were conducted from the healthcare payer perspective (66\%), whilst the remaining 16 adopted a societal perspective (33\%).
Twenty-seven studies (57\%) had a sample size smaller than 500 patients.

Different types of integrated care strategies were identified and grouped in integrated case management interventions $(n=10,21 \%)$, integrated care teams $(n=9,19 \%)$, coordination between different services $(n=7,15 \%)$, integrated care pathways $(n=4,9 \%)$ and integrated care programs based on disease management $(n=4,9 \%)$. The majority of the studies used care as usual as a comparison group (98\%), just one study estimated the cost-effectiveness of different levels of disease management intensity [24].

Principal outcomes extracted to assess effectiveness were quality of life (QOL) and quality-adjusted life-years (QALY), although several studies opted to measure mortality rates $[52,61,73,74]$ or other clinical performance scores [54, 57, 65, 68, 72] (Supplementary material Table S4). Economic results were largely reported as mean costs of the implemented integrated care program in comparison to the
Fig. 1 Updated flow chart of study selection

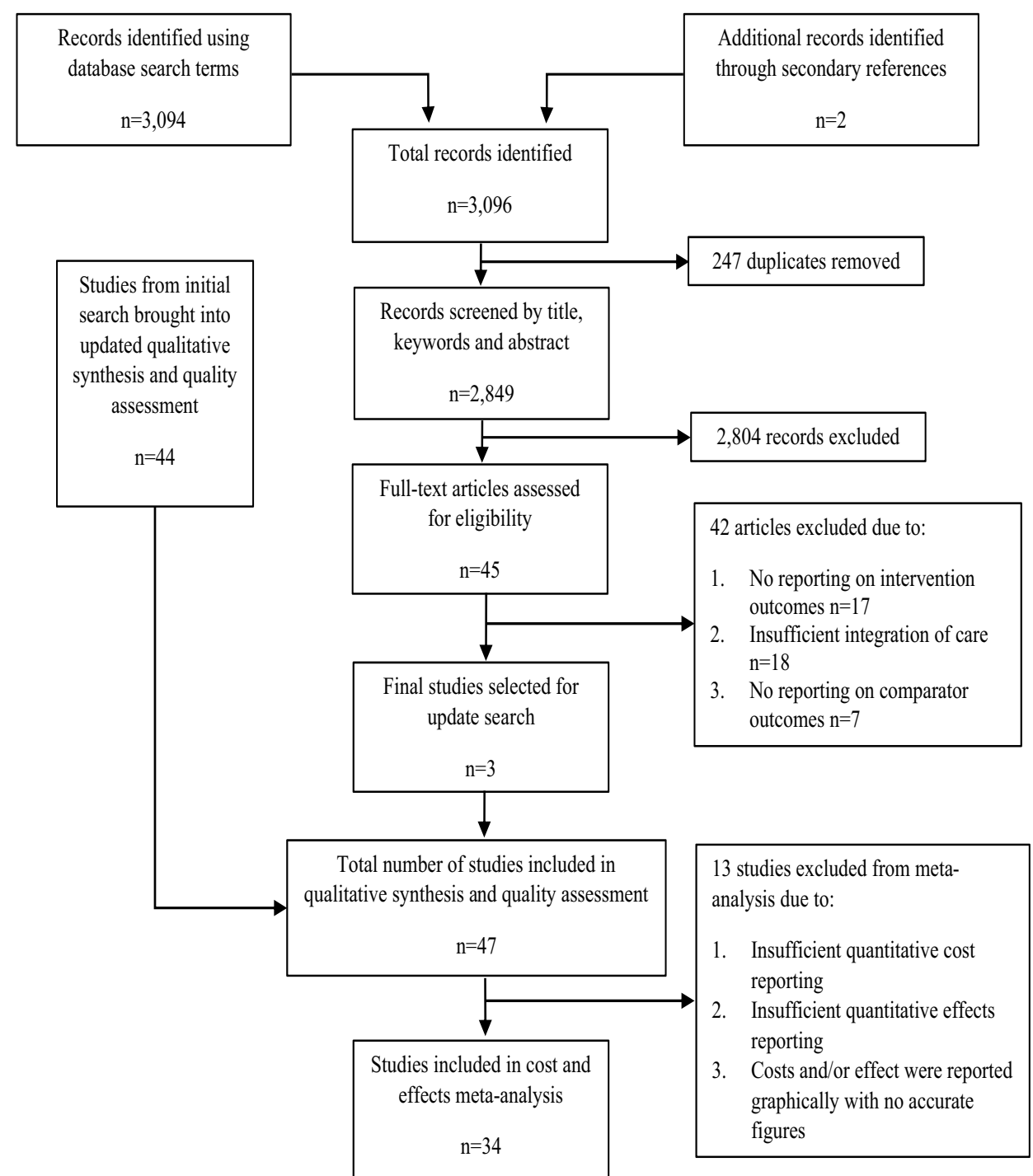


equivalent costs borne by the control group. Three studies $[52,55,64]$, however, reported the costs of the control and intervention groups after both a baseline period of 3 months and after implementation of the intervention programme. Similar baseline reporting practice was also undertaken in six studies with regards to the measuring of effectiveness $[36,64,68,69,71,74]$. In these cases, the final mean costs or outcomes of each group were adjusted (using Eq. 1 and 2 ) for their differing baseline values before entering the meta-analysis.

\section{Quality assessment}

The quality score of the 47 selected studies varied from $27.6 \%$ [43] to $96.7 \%$ [62] (Supplementary material Table S2) with a mean value of $65.6 \%( \pm 18.5)$. All studies had clear a description of the intervention strategy and outcomes measured. All but four studies featured an intervention and control group; the remaining studies instead used a pre-post comparison without a control group [40, 44, 63, 74]. Study populations were randomly allocated to either the control or intervention group in $60 \%$ of cases. Whilst most of the remaining $40 \%$ of studies used tools such as propensity score matching to adjust for differences between the intervention and control group, several observational studies were subject to selection bias and made no attempt to adjust for confounding factors at baseline. Although most studies defined their inclusion and exclusion criteria (87\%), 26\% did not provide information about any dropouts sustained during the study period. Furthermore, despite a large proportion $(85 \%)$ of the selected studies describing relevant aspects of the systems in which the intervention took place, only $36 \%$ of studies proceeded to take measures to avoid co-intervention or contamination within said system.

Descriptions of both outcome measures and sources of resource utilization were clear in $100 \%$ and $91 \%$ of studies respectively. The reporting of costs, however, was less promising and often exclusively covered direct medical costs of the intervention and control, while only $38 \%$ of selected studies included development and implementation costs of the intervention programme. In addition, only $24 \%$ of studies with time horizons over 12 months performed cost discounting [49, 58, 66, 75]. Nevertheless, cost and utilization across both social and health sectors were measured in $70 \%$ of selected studies.

Statistical analyses performed in the studies varied substantially in quality. Although relevant information of all parameters was reported in the vast majority of studies (91\%), 62\% dealt with missed observations [82] and 70\% appropriately handled skewed data [83]. Variability is inherent due to the nature of these study populations, particularly those providing interventions to larger sample sizes $[46,47,54,61,64,72-75,84]$. Despite this irregularity in baseline characteristics, most studies (51\%) did not perform sub-group analysis to examine heterogeneity of the results. Cost-effectiveness was analyzed using the incremental costeffectiveness ratio (ICER) method in $57 \%$ of the studies, while only $11 \%$ opted for either net-monetary or net-health benefit analysis $[70,72,76]$.

\section{Reported costs and effects}

Reported costs and outcomes are summarized in Supplementary material Tables S3 and S4. Studies included different types of costs in their total cost sum, including the following cost categories: inpatient, outpatient, development, implementation, societal, travel and productivity losses. Similarly, when reporting relative effectiveness, a variety of outcome measures were used across the studies. The outcomes observed included: quality of life (e.g., SF-12, EQ-5D, WHOQOLBRIEF), quality-adjusted life-years (QALYs), clinical outcomes, and mortality. Most economic evaluations (75\%) used QALY to measure health outcomes. Seventeen studies $(50 \%)$ reported healthcare cost savings, although this cost decrease in comparison to usual care was only significant in nine studies. Regarding outcomes, twenty-two studies (65\%) reported improved effectiveness in intervention groups. Of these, thirteen studies showed statistically significant improvement in outcomes compared to usual care.

\section{Results of main meta-analysis}

Figure $2 \mathrm{a}$ shows the results of the meta-analysis on healthcare utilization costs. After meta-analysis of cost ratios with appropriate quality score-based weighting, the overall ratio of mean costs was 0.944 (95\% CI 0.900-0.988) in favor of lower cost of the intervention group. The heterogeneity in ratio of mean costs across the studies was large $\left(I^{2}=97.6 \%\right)$. Although indicating a 5.6\% decrease in cost as a result of care integration, the cost reduction was borderline statistically significant. Figure $2 \mathrm{~b}$ illustrates the results of the meta-analysis of outcomes. The overall ratio of mean outcomes was 1.062 (95\% CI 1.048-1.077), suggesting a 6.2\% statistically significant improvement in outcomes associated with integrated care. However, the heterogeneity in the ratio of mean outcome across the studies was similarly large $\left(I^{2}=99.2 \%\right)$.

\section{Results of sub-group meta-analysis}

Results from subgroup meta-analysis by study-duration, -design, -region and -type of integrated care intervention are shown in Table 1 (forest plots are displayed in Supplementary material Figures S2a-d and S3a-d). The subgroup analysis showed that integrated care was associated with 


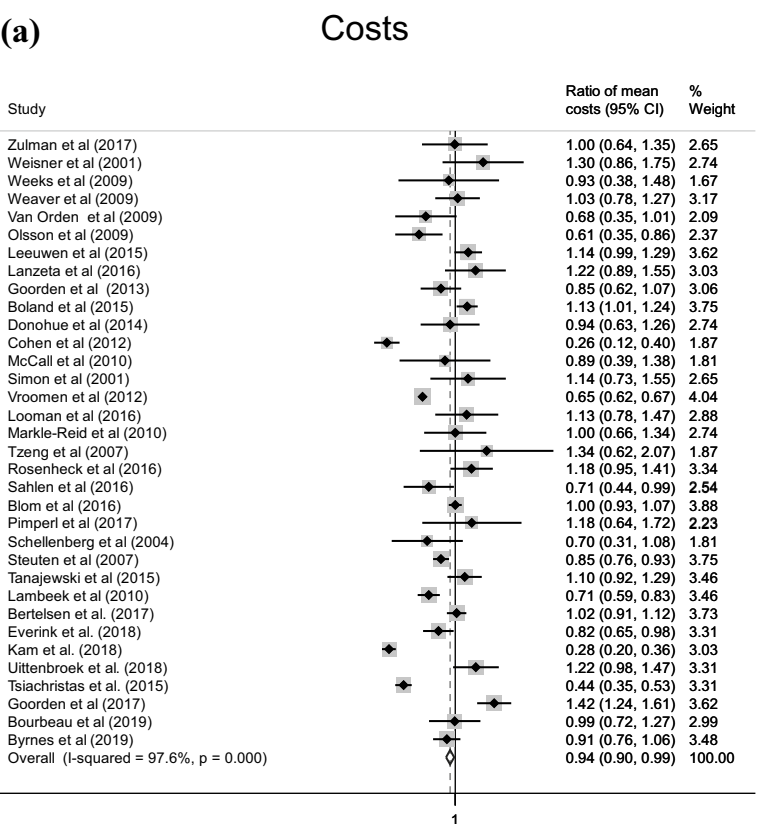

(b)

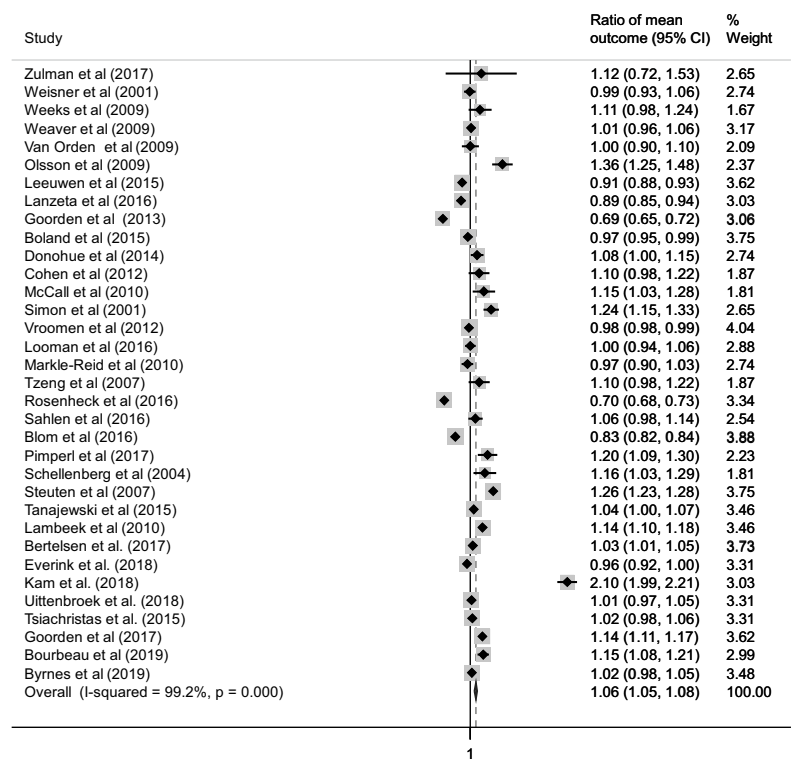

Fig. 2 Mean differences in $\mathbf{a}$ costs and $\mathbf{b}$ outcomes between the intervention and control groups. A larger format is attached in Supplementary material Figures $\mathrm{S} 2 / 3$

Table 1 Pooled results of the meta-analysis of healthcare costs and hospitalization costs by subgroups

\begin{tabular}{|c|c|c|c|c|}
\hline Characteristic & Subgroup & Study $(n)$ & $\begin{array}{l}\text { Mean weighted ratio of costs (mean } \\
\text { intervention cost/mean control cost) }\end{array}$ & $\begin{array}{l}\text { Mean weighted ratio of effects (mean } \\
\text { intervention effect/mean control effect) }\end{array}$ \\
\hline \multirow[t]{4}{*}{ Region } & North America & 10 & 1.000 (95\% CI 0.888-1.112) & 1.029 (95\% CI 0.981-1.077) \\
\hline & Europe & 20 & 0.951 (95\% CI 0.905-0.997) & 1.025 (95\% CI 1.015-1.035) \\
\hline & Australia/Asia & 3 & 0.778 (95\% CI 0.603-0.954) & 1.426 (95\% CI 1.376-1.477) \\
\hline & Africa & 1 & 0.695 (95\% CI 0.309-1.082) & 1.164 (95\% CI 1.035-1.294) \\
\hline \multirow[t]{3}{*}{ Study design } & $\mathrm{RCT}$ & 17 & 0.993 (95\% CI 0.930-1.057) & 1.093 (95\% CI 1.069-1.118) \\
\hline & Cluster RCT & 7 & 1.010 (95\% CI 0.933-1.087) & $0.922(95 \%$ CI $0.908-0.937)$ \\
\hline & Observational $^{\mathrm{a}}$ & 10 & 0.781 (95\% CI 0.687-0.874) & $1.126(95 \%$ CI $1.101-1.152)$ \\
\hline \multirow[t]{4}{*}{ Study duration } & $>12$ months & 12 & 0.868 (95\% CI 0.801-0.935) & 1.148 (95\% CI 1.112-1.184) \\
\hline & $7-12$ months & 14 & $0.980(95 \%$ CI $0.919-1.041)$ & 0.998 (95\% CI 0.985-1.011) \\
\hline & $\leq 6$ months & 7 & 1.002 (95\% CI 0.877-1.126) & 1.046 (95\% CI 1.022-1.070) \\
\hline & NA & 1 & $0.929(95 \%$ CI $0.383-1.475)$ & $1.110(95 \%$ CI $0.980-1.241)$ \\
\hline \multirow{5}{*}{$\begin{array}{l}\text { Type of integrated } \\
\text { care intervention }\end{array}$} & Integrated care team & 9 & 0.901 (95\% CI 0.824-0.979) & 0.961 (95\% CI 0.792-1.13) \\
\hline & Coordination between services & 7 & 1.160 (95\% CI 1.033-1.286) & 1.068 (95\% CI $1.044-1.092)$ \\
\hline & Integrated care management & 10 & 0.914 (95\% CI 0.817-1.012) & 1.178 (95\% CI 1.152-1.204) \\
\hline & Integrated care pathways & 4 & $0.922(95 \%$ CI $0.845-1.000)$ & 0.980 (95\% CI 0.955-1.004) \\
\hline & Disease management programs & 4 & 0.795 (95\% CI $0.716-0.874)$ & 1.114 (95\% CI 1.093-1.135) \\
\hline
\end{tabular}

${ }^{\mathrm{a}}$ Observational studies include; pre-post cohort, cohort, and cross-sectional studies

lower cost and higher outcomes across all regions, although this association was not always statistically significant. Studies originating from Australia/Asia indicated the largest cost savings (ratio of means: 0.778 ; 95\% CI $0.603-0.954$ ) and health benefit (ratio of means: 1.426; 95\% CI 1.376-1.477) followed by studies from Europe with ratio of mean costs of 0.951 (95\% CI 0.905-0.997) and ratio of mean outcome of 1.025 (95\% CI 1.015-1.035). Moreover, observational studies showed that integrated care was associated with lower cost (ratio of means: 0.781 ; 95\% CI $0.687-0.874$ ) and improvement in outcomes (ratio of means: 1.126; $95 \%$ CI 1.101-1.152). Neither the RCT nor Cluster RCT studies 
showed a significant association of integrated care with costs. However, RCTs showed a significant improvement in outcomes (ratio of means: 1.093; 95\% CI 1.069-1.118) and Cluster RCT studies showed a significant worsening of outcomes (ratio of means: 0.922; 95\% CI 0.908-0.937). Furthermore, studies lasting over 12 months showed a decrease in cost (ratio of means: 0.868 ; $95 \%$ CI 0.801 to 0.935 ) and improvement in outcomes (ratio of means: $1.148 ; 95 \%$ CI 1.112-1.184); whereas, these associations were not significant in studies with follow-up less than a year. Regarding the type of integrated care, the analysis reveals that disease management programs were associated with lower costs (ratio of means: 0.795 ; 95\% CI 0.716-0.874) and improvement in outcomes (ratio of means: 1.114; 95\% CI 1.093-1.135). The other types were associated only with either cost reduction (integrated care teams), cost increase (coordination between teams), or improved outcomes (integrated care management). Integrated care pathways showed no significant change in costs or effects.

\section{Discussion}

\section{Findings and interpretation}

This is the first meta-analysis of economic evaluations of integrated care across different clinical and care areas as well as types of integration. The results indicate that integrated care was associated with lower costs and improved outcomes compared with usual care, especially in studies with a follow-up period over a year. This may reflect the need for a sufficiently long follow-up period for effects to emerge, especially if there is a learning period following implementation [85]. In addition, studies with an extended follow-up period are more likely to capture long-term reductions in cost that may negate and surpass the initial investment in developing and implementing integrated care [86].

Moreover, our results raise questions about whether the long-term impact of integrated care has been captured in the economic evaluations. Decision analytic modeling that extrapolates costs and outcomes beyond study follow-up is recommended, especially when benefits of deliberating treatment plans for chronic diseases may be ongoing [28]. Nevertheless, it was performed in only one study [43]. Similarly, integrated care inherently intersects care boundaries and impacts a broad range of costs and outcomes within and beyond the healthcare system. However, only about a third of the economic evaluations included an (sensitivity) analysis taking the societal perspective, potentially missing more widespread cost savings of integrated care interventions related to costs in other sectors, informal care-giving, and productivity [87]. Taking the societal perspective in economic evaluations of integrated care is demanding and requires more complex and costly data collection. Although a health payer perspective remains the approach recommended by bodies such as the National Institute for Health and Care Excellence (NICE), this may jeopardize the quality of evidence about the cost-effectiveness of integrated care.

The pooled results of observational economic evaluations showed both significant reduction in costs and improvement in outcomes. However, this evidence was not found in studies with experimental designs. This contrast in the findings by study design highlights a well-documented trade-off between attributability and practicability [88]. In our review, several observational economic evaluations barely took any measures to mitigate for treatment contamination or selection bias, thereby jeopardizing causal inference $[36,52,55$, $64,68,69,71,74]$. Although experimental designs are the gold standard for robust causal inference, their adoption in evaluating integrated care has been criticized due to their rigidness and low generalizability $[89,90]$.

Studies from Europe and Australia/Asia were significant in both costs and outcomes; whereas, studies from North America showed no significant effects. The reasons for this are unclear but could be owing to differences in healthcare systems [91] or the stage of implementation of integrated care interventions. North America is at a more advanced stage; it is possible, therefore, that the studies implemented are broader and involve larger populations, which may dilute the effects relative to smaller studies elsewhere where integrated care is still at a nascent stage [18].

Among types of intervention, disease management interventions alone showed significant decreases in costs and improvements in outcomes. This is similar to the findings of previous meta-analyses of disease management programs on single chronic conditions $[20,92,93]$ and may mean that integrated care interventions are implemented more easily within single disease areas. Indeed, many initiatives around the world have started integrating services within single chronic conditions as a first step towards a wider integration [17]. Disease management programs have been long implemented in North America and Europe and certain levels of efficiency may have been achieved due to experience and productivity [94]. However, an important challenge remains, since disease management programs may not meet the needs of a patient with multiple health problems with complex needs [95].

\section{Quantity and quality of economic evaluations}

Substantial investment into the implementation of integrated care is occurring on a global scale [96]. Despite this, only 34 economic evaluations of integrated care were identified in this review that had sufficient reported costs and outcomes to be included in the meta-analysis. Of these studies, only 19 (56\%) had a quality score over $70 \%$ - a score above which is 
generally given to a study of "fair standard" [97]. The relatively low number of economic evaluations and their moderate methodological quality may stem from two reasons. First, economic evaluations are increasingly piggy backing effectiveness assessments of integrated care and are subject to insufficient communication between health economists and clinical/health service researchers [88]. Hence, regarding economic evaluations as an "afterthought" may be contributing to such a remarkably low number of suitable studies for meta-analysis. Second, integrated care interventions, like many complex interventions, are frequently not subject to extensive health technology assessment (HTA) as part of a reimbursement process at the national level. As a result, the cost-effectiveness of integrated care may receive less scrutiny than other health interventions (e.g., pharmaceuticals and clinical technologies) traditionally subject to HTA.

\section{Limitations}

First, although the search strategy deployed aimed to include all studies broadly fitting the pre-set definition of integrated care, this review may have missed studies characterized by an alternative approach not covered by the search terms. Therefore, this review and meta-analysis may exclude some economic evaluations of interventions that could broadly fall under the integrate care umbrella term without explicitly fitting our working definition. However, it is expected that this may be the case for only a few studies as we have used broad concepts of integrated care in our search strategy. Second, despite the use of a binary system to review and assign quality scores to each economic evaluation based on the CHEERSadapted checklist, there was opportunity for subjectivity which may have biased the scoring. Finally, due to the lack of reported standard error/ deviation of mean costs and outcomes, the heterogeneity across the studies reflected in the $I^{2}$ statistic was based on the study quality rather than the precision of the mean [98]. Therefore, the statistical significance of the meta-analysis results should be interpreted with caution.

\section{Policy and research implications}

Our findings support the reorientation of healthcare systems towards integration of care to help policy makers to meet increased demand for health and social care within tight budgets. However, with such sparse economic evaluations in integrated care, there is insufficient evidence about the factors that determine the cost-effectiveness of integrated care, such as models of care integration, implementation process, and target population. Efficiency of research is being streamlined in most other health innovations (e.g., pharmaceuticals and medical technologies) by including them in reimbursement processes with cost-effectiveness as an explicit criterion for market access [99]. Similar efforts should be made in assessing the cost-effectiveness of integrated care. Measures could be taken to standardize description of intervention and comparator, reporting of methods and results; apply appropriate follow-up periods and decision-analytic models; address bias; and deploy explicit decision criteria when value-formoney is uncertain. Such directed expansion of health economics towards the evaluation of integrated care is necessary to ensure decisions surrounding the implementation of integrated healthcare delivery are likely to benefit, rather than hinder, aims to meet increasing demands on tightening budgets.

Open Access This article is licensed under a Creative Commons Attribution 4.0 International License, which permits use, sharing, adaptation, distribution and reproduction in any medium or format, as long as you give appropriate credit to the original author(s) and the source, provide a link to the Creative Commons licence, and indicate if changes were made. The images or other third party material in this article are included in the article's Creative Commons licence, unless indicated otherwise in a credit line to the material. If material is not included in the article's Creative Commons licence and your intended use is not permitted by statutory regulation or exceeds the permitted use, you will need to obtain permission directly from the copyright holder. To view a copy of this licence, visit http://creativecommons.org/licenses/by/4.0/.

\section{References}

1. Lorenzoni, L. et al.: Health spending projections to 2030: new results based on a revised OECD methodology, in OECD Health Working Papers. In: Publishing, O. (ed.) Paris (2019)

2. Whitty, C.J.M., et al.: Rising to the challenge of multimorbidity. BMJ 368, 16964 (2020)

3. Improvement, N.: Performance of the NHS provider sector for the year ended 31 March 2018. NHS Improvement, London (2018)

4. van Vliet, K., Oudenampsen, D.: Integrated care in the Netherlands. Verwey-Jonker Instituut. (2004)

5. Åhgren, B.: Chain of care development in Sweden: results of a national study. Int. J. Integr. Care. 3 (2003)

6. Plochg, T., Klazinga, N.S., Starfield, B.: Transforming medical professionalism to fit changing health needs. BMC Med. 7(1), 64 (2009)

7. Shaw, S., Rosen, R., Rumbold, B.: What is integrated care, p. 7. Nuffield Trust, London (2011)

8. Allen, D., Gillen, E., Rixson, L.: The effectiveness of integrated care pathways for adults and children in health care settings: a systematic review. JBI Database Syst. Rev. Implement. Rep. 7(3), 80-129 (2009)

9. OECD: Delivering person-centred integrated care: synthesis of case studies from OECD health systems. OECD, Paris (2015)

10. Stein, K.V. Rieder, A.: Integrated care at the crossroads-defining the way forward. Int. J. Integr. Care. 9 (2009)

11. Hildebrandt, H., Schulte, T., Stunder, B.: Triple aim in Kinzigtal, Germany: improving population health, integrating health care and reducing costs of care-lessons for the UK? J. Integr. Care. 20(4), 205-222 (2012)

12. Berwick, D.M., Nolan, T.W., Whittington, J.: The triple aim: care, health, and cost. Health Aff. 27(3), 759-769 (2008)

13. Coulter, A.: Shifting the balance from secondary to primary care. Br. Med. J. Publ. Group (1995) 
14. Baxter, S., et al.: The effects of integrated care: a systematic review of UK and international evidence. BMC Health Serv. Res. 18(1), 350 (2018)

15. Looman, W.M., Huijsman, R., Fabbricotti, I.N.: The (cost-) effectiveness of preventive, integrated care for community-dwelling frail older people: a systematic review. Health Soc. Care. Commun. 27(1), 1-30 (2019)

16. Damery, S., Flanagan, S., Combes, G.: Does integrated care reduce hospital activity for patients with chronic diseases? An umbrella review of systematic reviews. BMJ open 6(11), e011952 (2016)

17. Nolte, E., Pitchforth, E.: What is the evidence on the economic impacts of integrated care? (2014)

18. Kadu M, E.N., Stein V, Tsiachristas A.: Methodological quality of economic evaluations in integrated care: evidence from a systematic review. Int. J. Integr. Care. (2019)

19. Mounier-Jack, S., Mayhew, S.H., Mays, N.: Integrated care: learning between high-income, and low- and middle-income country health systems. Health Policy Plan 32(suppl_4), iv6-iv12 (2017)

20. Boland, M.R., et al.: The health economic impact of disease management programs for COPD: a systematic literature review and meta-analysis. BMC Pulmonary. Med. 13(1), 40 (2013)

21. Desmedt, M., et al.: Economic impact of integrated care models for patients with chronic diseases: a systematic review. Value Health 19(6), 892-902 (2016)

22. International Care Foundation. "Integrated Care search". https:// integratedcarefoundation.org/ific-integrated-care-search. (2019)

23. Siaw, M., et al.: Cost-effectiveness of multidisciplinary collaborative care versus usual care in the management of high-risk patients with diabetes in Singapore: short-term results from a randomized controlled trial. J. Clin. Pharm. Ther. 43(6), 775-783 (2018)

24. Tsiachristas, A., Burgers, L., Rutten-van Mölken, M.P.: Costeffectiveness of disease management programs for cardiovascular risk and COPD in the Netherlands. Value Health 18(8), 977-986 (2015)

25. Oordt-Speets, A.M., et al.: Global etiology of bacterial meningitis: a systematic review and meta-analysis. PLoS ONE 13(6), e0198772 (2018)

26. Sayers, A.: Tips and tricks in performing a systematic review. Br. J. Gen. Pract. 57(545), 999 (2007)

27. Kodner, D.L., Spreeuwenberg, C.: Integrated care: meaning, logic, applications, and implications-a discussion paper. Int. J. Integr. Care 2 (2002)

28. Drummond, M.F., et al.: Methods for the economic evaluation of health care programmes. Oxford university press. (2015)

29. Huang, X., Lin, J., Demner-Fushman, D.: Evaluation of PICO as a knowledge representation for clinical questions. In AMIA annual symposium proceedings. American Medical Informatics Association. (2006)

30. Husereau, D., et al.: Consolidated health economic evaluation reporting standards (CHEERS) statement. Cost. Eff. Resour. Alloc. 11(1), 6 (2013)

31. Steuten, L.M.G., et al.: The health technology assessment-disease management instrument reliably measured methodologic quality of health technology assessments of disease management. J. Clin. Epidemiol. 57(9), 881-888 (2004)

32. Tsiachristas, A., et al.: Cost-effectiveness of strategies to increase cervical screening uptake at first invitation (STRATEGIC). J. Med. Screen. 25(2), 99-109 (2018)

33. Doi, S.A., Barendregt, J.J., Mozurkewich, E.L.: Meta-analysis of heterogeneous clinical trials: an empirical example. Contemp. Clin. Trials 32(2), 288-298 (2011)

34. Murad, M.H., et al.: When continuous outcomes are measured using different scales: guide for meta-analysis and interpretation. BMJ 364, k4817 (2019)
35. DerSimonian, R., Laird, N.: Meta-analysis in clinical trials. Control. Clin. Trials 7(3), 177-188 (1986)

36. Goorden, M., et al.: Cost-utility of collaborative care for the treatment of comorbid major depressive disorder in outpatients with chronic physical conditions. A randomized controlled trial in the general hospital setting (CC-DIM). Neuropsychiatr. Dis. Treat. 13, 1881-1893 (2017)

37. Byrnes, J., et al.: Within trial cost-utility analysis of disease management program for patients hospitalized with atrial fibrillation: results from the SAFETY trial. J Med. Econ. 22(9), 945-952 (2019)

38. Bourbeau, J., et al.: Cost-effectiveness of the COPD patient management European trial home-based disease management program. Int. J. Chron. Obstruct. Pulmon. Dis. 14, 645-657 (2019)

39. Dubuc, N., et al.: Meeting the home-care needs of disabled older persons living in the community: does integrated services delivery make a difference? BMC Geriatr. 11, 67 (2011)

40. Salmon, R.B., et al.: A collaborative accountable care model in three practices showed promising early results on costs and quality of care. Health Aff. (Millwood) 31(11), 2379-2387 (2012)

41. Celano, C.M., et al.: Cost-effectiveness of a collaborative care depression and anxiety treatment program in patients with acute cardiac illness. Value Health 19(2), 185-191 (2016)

42. Pozzilli, C., et al.: Home based management in multiple sclerosis: results of a randomised controlled trial. J. Neurol. Neurosurg. Psychiatry 73(3), 250-255 (2002)

43. Bergmann, J.N., et al.: Outcomes and cost-effectiveness of integrating HIV and nutrition service delivery: pilots in Malawi and Mozambique. AIDS Behav. 21(3), 703-711 (2017)

44. Koch, T.G., Wendling, B.W., Wilson, N.E.: How vertical integration affects the quantity and cost of care for Medicare beneficiaries. J. Health. Econ. 52, 19-32 (2017)

45. Bird, S.R., et al.: Integrated care facilitation model reduces use of hospital resources by patients with pediatric asthma. J. Healthc. Qual. 34(3), 25-33 (2012)

46. Goltz, L., et al.: Evaluation of a program of integrated care to reduce recurrent osteoporotic fractures. Pharmacoepidemiol. Drug. Saf. 22(3), 263-270 (2013)

47. Wiley-Exley, E., et al.: Cost-effectiveness of integrated care for elderly depressed patients in the PRISM-E study. J. Ment. Health Policy. Econ. 12(4), 205-213 (2009)

48. Karow, A., et al.: Cost-effectiveness of 12-month therapeutic assertive community treatment as part of integrated care versus standard care in patients with schizophrenia treated with quetiapine immediate release (ACCESS trial). J. Clin. Psychiatry 73(3), e402-e408 (2012)

49. Renaud, A., et al.: The cost effectiveness of integrated care for people living with HIV including antiretroviral treatment in a primary health care centre in Bujumbura Burundi. AIDS Care 21(11), 1388-1394 (2009)

50. Camacho, E.M., et al.: Long-term clinical and cost-effectiveness of collaborative care (versus usual care) for people with mentalphysical multimorbidity: cluster-randomised trial. Br. J. Psychiatry 213(2), 456-463 (2018)

51. Wise, C.G., et al.: Population-based medical and disease management: an evaluation of cost and quality. Dis. Manag. 9(1), 45-55 (2006)

52. Zulman, D.M., et al.: Effect of an intensive outpatient program to augment primary care for high-need veterans affairs patients: a randomized clinical trial. JAMA Intern. Med. 177(2), 166-175 (2017)

53. Weisner, C., et al.: Integrating primary medical care with addiction treatment: a randomized controlled trial. JAMA 286(14), $1715-1723$ (2001) 
54. Weeks, W.B., et al.: Higher health care quality and bigger savings found at large multispecialty medical groups. Health Aff. (Millwood) 29(5), 991-997 (2010)

55. Weaver, M.R., et al.: Cost-effectiveness analysis of integrated care for people with HIV, chronic mental illness and substance abuse disorders. J. Ment. Health. Policy. Econ. 12(1), 33-46 (2009)

56. van Orden, M., et al.: Collaborative mental health care versus care as usual in a primary care setting: a randomized controlled trial. Psychiatr Serv 60(1), 74-79 (2009)

57. Olsson, L.E., et al.: A cost-effectiveness study of a patient-centred integrated care pathway. J. Adv. Nurs. 65(8), 1626-1635 (2009)

58. van Leeuwen, K.M., et al.: Cost-effectiveness of a chronic care model for frail older adults in primary care: economic evaluation alongside a stepped-wedge cluster-randomized trial. J. Am. Geriatr. Soc. 63(12), 2494-2504 (2015)

59. Lanzeta, I., Mar, J., Arrospide, A.: Cost-utility analysis of an integrated care model for multimorbid patients based on a clinical trial. Gac. Sanit. 30(5), 352-358 (2016)

60. Goorden, M., et al.: Cost-utility analysis of a collaborative care intervention for major depressive disorder in an occupational healthcare setting. J. Occup. Rehabil. 24(3), 555-562 (2014)

61. Boland, M.R., et al.: Cost-effectiveness of integrated COPD care: the RECODE cluster randomised trial. BMJ Open 5(10), e007284 (2015)

62. Donohue, J.M., et al.: Twelve-month cost-effectiveness of telephonedelivered collaborative care for treating depression following CABG surgery: a randomized controlled trial. Gen. Hosp. Psychiatry 36(5), 453-459 (2014)

63. Cohen, E., et al.: Integrated complex care coordination for children with medical complexity: a mixed-methods evaluation of tertiary care-community collaboration. BMC Health Serv. Res. 12, 366 (2012)

64. McCall, N., Cromwell, J., Urato, C.: Evaluation of medicare care management for high cost beneficiaries (CMHCB) demonstration: Massachusetts general hospital and Massachusetts general physicians organization $(\mathrm{MGH})$, in research triangle park. RTI International, Baltimore (2010)

65. Simon, G.E., et al.: Cost-effectiveness of a collaborative care program for primary care patients with persistent depression. Am. J. Psychiatry 158(10), 1638-1644 (2001)

66. MacNeil Vroomen, J.L., et al.: Is it time for a change? A cost-effectiveness analysis comparing a multidisciplinary integrated care model for residential homes to usual care. PLoS ONE 7(5), e37444 (2012)

67. Looman, W.M., et al.: Cost-effectiveness of the 'Walcheren Integrated Care Model' intervention for community-dwelling frail elderly. Fam. Pract. 33(2), 154-160 (2016)

68. Markle-Reid, M., et al.: The effects and costs of a multifactorial and interdisciplinary team approach to falls prevention for older home care clients 'at risk' for falling: a randomized controlled trial. Can. J. Aging. 29(1), 139-161 (2010)

69. Tzeng, D.S., et al.: Healthcare in schizophrenia: effectiveness and progress of a redesigned care network. BMC Health Serv. Res. 7, 129 (2007)

70. Rosenheck, R., et al.: Cost-effectiveness of comprehensive, integrated care for first episode psychosis in the NIMH RAISE early treatment program. Schizophr Bull 42(4), 896-906 (2016)

71. Sahlen, K.G., Boman, K., Brannstrom, M.: A cost-effectiveness study of person-centered integrated heart failure and palliative home care: based on a randomized controlled trial. Palliat. Med. 30(3), 296-302 (2016)

72. Blom, J., et al.: Effectiveness and cost-effectiveness of a proactive, goal-oriented, integrated care model in general practice for older people. A cluster randomised controlled trial: integrated systematic care for older people - the ISCOPE study. Age Ageing 45(1), 30-41 (2016)
73. Pimperl, A., et al.: Evaluating the impact of an accountable care organization on population health: the Quasi-experimental design of the German Gesundes Kinzigtal. Popul. Health. Manag. 20(3), 239-248 (2017)

74. Armstrong Schellenberg, J.R., et al.: Effectiveness and cost of facility-based integrated management of childhood illness (IMCI) in Tanzania. Lancet 364(9445), 1583-1594 (2004)

75. Steuten, L., et al.: Cost-utility of a disease management program for patients with asthma. Int. J. Technol. Assess. Health. Care. 23(2), 184-191 (2007)

76. Tanajewski, L., et al.: Cost-effectiveness of a specialist geriatric medical intervention for frail older people discharged from acute medical units: economic evaluation in a two-centre randomised controlled trial (AMIGOS). PLoS ONE 10(5), e0121340 (2015)

77. Bertelsen, J.B., et al.: Shared care versus hospital-based cardiac rehabilitation: a cost-utility analysis based on a randomised controlled trial. Open Heart 5(1), e000584 (2018)

78. Everink, I.H.J., et al.: An economic evaluation of an integrated care pathway in geriatric rehabilitation for older patients with complex health problems. PLoS ONE 13(2), e0191851 (2018)

79. Wong, F.K.Y., et al.: Cost-effectiveness of a transitional home-based palliative care program for patients with end-stage heart failure. Palliat. Med. 32(2), 476-484 (2018)

80. Uittenbroek, R.J., et al.: Integrated and person-centered care for community-living older adults: a cost-effectiveness study. Health Serv. Res. 53(5), 3471-3494 (2018)

81. Stovold, E., et al.: Study flow diagrams in Cochrane systematic review updates: an adapted PRISMA flow diagram. Syst. Rev. 3, 54 (2014)

82. Faria, R., et al.: A guide to handling missing data in cost-effectiveness analysis conducted within randomised controlled trials. Pharmacoeconomics 32(12), 1157-1170 (2014)

83. Malehi, A.S., Pourmotahari, F., Angali, K.A.: Statistical models for the analysis of skewed healthcare cost data: a simulation study. Health Econ. Rev. 5, 11 (2015)

84. Tsiachristas, A., Burgers, L., Rutten-van Molken, M.P.: Cost-Effectiveness of disease management programs for cardiovascular risk and COPD in The Netherlands. Value Health 18(8), 977-986 (2015)

85. Meacock, R.: Methods for the economic evaluation of changes to the organisation and delivery of health services: principal challenges and recommendations. Health Econ. Policy. Law. 14(1), 119-134 (2019)

86. Serxner, S., K. Baker, and D. Gold, Guidelines for analysis of economic return from health management programs. Am J Health Promot, 2006. 20(6): p. suppl 1-17.

87. Wilkinson, J.R., Murray, S.A.: Assessment in primary care: practical issues and possible approaches. BMJ 316(7143), 1524-1528 (1998)

88. Tsiachristas, A., et al.: Performing economic evaluation of integrated care: highway to hell or stairway to heaven? Int. J. Integr. Care. 16(4), 3 (2016)

89. Tsiachristas, A., Rutten-van Mölken, M.: Evaluating complex interventions, in Handbook Integrated Care. In: Amelung, V.E., et al. (eds.) Springer International Publishing (2017)

90. Hawe, P., Shiell, A., Riley, T.: Complex interventions: how "out of control" can a randomised controlled trial be? BMJ 328(7455), 1561-1563 (2004)

91. Calciolari, S., Ilinca, S.: Lessons from international experiences: comparative case studies of integrated care in Europe and North America. Int. J. Integr. Care 11 (2011)

92. Krause, D.S.: Economic effectiveness of disease management programs: a meta-analysis. Dis. Manag. 8(2), 114-134 (2005)

93. Neumeyer-Gromen, A., et al.: Disease management programs for depression: a systematic review and meta-analysis of randomized controlled trials. Med. Care. 42(12), 1211-1221 (2004)

94. Tsiachristas, A.: Payment and economic evaluation of integrated care. Int. J. Integr. Care. 15, e013 (2015) 
95. Nolte, E., McKee, M.: Caring for people with chronic conditions. A health system perspective. Open University Press. (2008)

96. Scheller-Kreinsen, D., Blümel, M., Busse, R.: Chronic disease management in Europe. Eurohealth. 15(1)

97. Nguyen, H.N., Ly, K.N., Vo, Q.T.: Assessing the quality of health economic evaluation research by cheers instrument: a critical literature review in Laos, Cambodia, And Myanmar. J. Appl. Pharm. Sci. 7(6), 222-228 (2017)

98. Higgins, J.P., et al.: Measuring inconsistency in meta-analyses. BMJ 327(7414), 557-560 (2003)
99. National Institute for Health and Clinical Excellence, Research Recommendations Interim Process and methods guide. (2015)

Publisher's Note Springer Nature remains neutral with regard to jurisdictional claims in published maps and institutional affiliations. 